\title{
Mathematical modeling and experimental breakthrough curves of cephalosporin $C$ adsorption in a fixed-bed column
}

\author{
Carlos André V. Burkerta ${ }^{\mathrm{a}}$, Geraldo N.O. Barbosa ${ }^{\mathrm{c}}$, Marcio A. Mazutti ${ }^{\mathrm{b}, *}$, Francisco Maugeri ${ }^{\mathrm{c}}$ \\ a Escola de Química e Alimentos, Universidade Federal do Rio Grande, Rua Engenheiro Alfredo Huch, 475, P.O. Box 474, Rio Grande, RS, Brazil \\ ${ }^{\mathrm{b}}$ Department of Chemical Engineering, Federal University of Santa Maria - UFSM, Av. Roraima, 1000, CEP 97105-900, Santa Maria, RS, Brazil \\ c Department of Food Engineering, University of Campinas, P.O. Box 6121, CEP 13083-862, Campinas, SP, Brazil
}

\section{A R T I C L E I N F O}

\section{Article history:}

Received 10 June 2010

Received in revised form 10 February 2011

Accepted 16 February 2011

\section{Keywords:}

Cephalosporin C

Mathematical modeling

Particle Swarm Optimization

Adsorption

\begin{abstract}
A B S T R A C T
This work presents the mathematical modeling of the cephalosporin C(CPC) adsorption process in a fixedbed column. The application of Particle Swarm Optimization (PSO) algorithm for parameter estimation was first considered, which shows to be a useful tool for parameter estimation in adsorption processes. Modeling and simulation of CPC purification showed a good performance during both estimation and validation step. After this, a central composite rotational design (CCRD) was conceived taking into account both the superficial velocity of liquid and column length as process variables with the responses obtained from the application of the model, which works as a process simulator affording a process answer from a given input. It has been shown that the response surface methodology was an efficient optimization tool, since few simulations were required to find the optimum region for independent variables.
\end{abstract}

(C) 2011 Elsevier Ltd. All rights reserved.

\section{Introduction}

The commercialization of products obtained from biotechnological processes requires a coordinated coupling of unit operations in order to develop an efficient process. In most of these biotechnological processes, the main product of interest is generally synthesized along with other byproducts showing analogous chemical and physical properties. Thus, the need for more efficient downstream processing is essential to decrease the final costs of those biotechnological products. Adsorption processes are widely used unit operations in downstream processing of many products obtained by fermentation, including cephalosporin $C$ (CPC), which is a $\beta$ lactamic antibiotic precursor that is the starting raw material for the synthesis of a wide-ranging spectrum of bactericidal antibiotics [1].

A large-scale cyclic operation involves three steps: adsorption, desorption and washing. Design and scale-up of such large-scale separation process require basic information to simulate the dynamic behavior in both adsorption and desorption steps [2]. The model can be formulated on the basis of conservation equations, mass transport within the resin particles, and equilibrium relationship at the liquid-solid interface. The model parameters such as equilibrium data, film mass transfer coefficient, intraparticle diffusivity and axial dispersion can be measured experimentally, or predicted by existing correlation, or optimized from experimental data [3].

\footnotetext{
* Corresponding author. Tel.: +55 5532209591 .

E-mail address: marciomazutti@gmail.com (M.A. Mazutti).
}

The usefulness and confidence of the model predictions are dependent of the quality of the estimated parameters. The use of empirical correlation to estimate mass transport parameter imposes restrictions to the model. If the values of real parameters are out of the range in what the correlation is valid the model predictions are failure. Regarding the equilibrium parameters, it is preferable to estimate their values from process data instead of equilibrium data because some imprecision during the estimation procedure probably will compromise the overall model performance. The most published works in the current literature regarding the mathematical modeling of adsorption process of CPC are referring the use of empirical correlations or equilibrium data to estimate the model parameters [3-5].

Ideally, if the experiments, whose data are used during the parameter estimation procedure, were correctly designed, a unique set of parameters exists and the global optimum for the system is easily determined by appropriated optimization routines. Otherwise, it implicates in failure to fit the experimental data. There are several optimization routines available to estimate the model parameters, such as Particle Swarm Optimization (PSO) [6,7], simulated annealing [8], ant algorithm [9] and genetic algorithm [10].

The PSO is a heuristic algorithm based on the social behavior of collection of animals. Each individual of the swarm, called particle, remembers the best solution found by itself and by the whole swarm along the search trajectory. The PSO presents interesting characteristics along the iterations. In the initial iterations, the random character of the search is high and the particles conduct a global search over search region. As the iterations evolve, the particles concentrate around the more promising regions found during 
Table 1

Typical physical properties of the Amberlite XAD-2 resin.

\begin{tabular}{ll}
\hline Physical properties & Corresponding values \\
\hline Solids (\%) & 55.0 \\
Porosity (pore $\mathrm{mL}^{-1}$ bead) & 0.42 \\
Surface area $\left(\mathrm{m}^{2} \mathrm{~g}^{-1}\right)$ & 300.0 \\
Mean pore diameter $(\AA \hat{)})$ & 90.0 \\
Bulk density $\left(\mathrm{g} \mathrm{L}^{-1}\right)$ & 640.0 \\
True wet density $\left(\mathrm{g} \mathrm{mL}^{-1}\right)$ & 1.02 \\
Skeletal density $\left(\mathrm{g} \mathrm{mL}^{-1}\right)$ & 1.07 \\
Particle size range $(\mu \mathrm{m})$ & $210-500$ \\
\hline
\end{tabular}

the exploration stage [7]. PSO was previously reported as good method for parameter estimation in adsorption processes for separation of enzyme in expanded-bed column [11], biosorption of synthetic dyes from water [12] and separation of sugars from the reaction mixture for production of fructooligosaccharides using the $\mathrm{X}$ zeolite as adsorbent in stirred-tank reactor [13].

In this context, the main objective of this work is modeling of cephalosporin C adsorption in a fixed-bed column. Breakthrough curves at several experimental conditions were first carried out aiming the collection of experimental data to estimate the model parameters by the PSO algorithm. Afterwards, the validated model was used as a process simulator and the response surface methodology was employed to optimize the column efficiency and breakthrough time.

\section{Materials and methods}

\subsection{Chemicals}

A nonspecific polystyrene macroporous adsorbent, Amberlite XAD-2 (Rohm \& Haas Company), was used for evaluating the influence of geometric and operational column parameters on the performance of the separation of cephalosporin $\mathrm{C}$. The properties of the Amberlite XAD-2 resin are described in Table 1.

\subsection{Apparatus, process start-up and analytical methods}

Amberlite XAD-2 was sieved to obtain an adequate fraction of particle sizes with $0.210 \mathrm{~mm}, 0.297 \mathrm{~mm}$ and 0.500 mesh screens. For removal of small particles, the resin was washed with methanol at a Soxhlet extractor [14,15], followed by washing with formic acid buffer $\mathrm{pH}$ 3.6, prepared as for removal of methanol [16].

The studies of separation of cephalosporin C were carried out in Glass Columns C 10/20 from Pharmacia Biotech (Uppsala, Sweden), $200 \mathrm{~mm}$ in length and $10 \mathrm{~mm}$ internal diameter, containing pretreated resin Amberlite XAD-2. The jacketed glass column was maintained at temperature of $25^{\circ} \mathrm{C}$ using water circulation bath. For each test the column was equilibrated by passing a buffer solution of formic acid ( $\mathrm{pH}$ 3.6) in volume equivalent to five times the volume of the bed of particles. Detection of cephalosporin $C$ concentration effluent from the column was performed by UV spectrophotometer at $260 \mathrm{~nm}$ (Micronal, B-382).

Each experiment was carried out until the output concentration remained constant for $2 \mathrm{~h}$. All the experiments were conducted in duplicate and the error is smaller than 3.5\%. The conditions used in the experiments are shown in Table 2.

\section{Model formulation}

The development of a mathematical model for the CPC adsorption process in a fixed bed column packed with the Amberlite
XAD-2 resin was based on the following hypotheses:

i. The column presents a homogenous packing.

ii. The spherical resin was considered a opaque solid, assuming that there are no internal CPC diffusion.

iii. Instantaneous equilibrium between the solid and liquid phase concentrations at the external surface of the particles.

iv. The hydrodynamic behavior of the liquid phase could be described by the axial dispersion model.

v. Isothermal adsorption column. After a preliminary screening amongst the isotherm models of Langmuir, Freudlich, linear and BET, it was verified that the Langmuir model is the most suitable to represent the equilibrium data for the CPC adsorption.

vi. The rheological properties of the bulk phase were considered to be the same in all the experiments, and independent of the CPC concentration.

The differential mass balance in a volume element of the column for CPC concentration may be expressed by:

$\frac{\partial C}{\partial t}=D_{L} \cdot \frac{\partial^{2} C}{\partial z^{2}}-v_{z} \cdot \frac{\partial C}{\partial z}-\left(\frac{1-\varepsilon}{\varepsilon}\right) \cdot \frac{3 \cdot k_{f}}{R_{p}} \cdot\left(C-C_{r=R_{p}}\right)$

where $D_{L}$ is the axial dispersion coefficient $\left(\mathrm{m}^{2} \mathrm{~h}^{-1}\right), k_{f}$ is the liquid film mass transfer coefficient $\left(\mathrm{m} \mathrm{h}^{-1}\right), v_{z}$ is the interstitial velocity $\left(\mathrm{m} \mathrm{h}^{-1}\right), R_{p}$ is the resin radius $(\mathrm{m}), \varepsilon$ is the bed porosity, $t$ is time $(\mathrm{h}), z$ is the axial axis (m), $C$ and $C_{r=R_{p}}$ are the CPC concentration in the bulk fluid and on the resin surface $\left(\mathrm{mg} \mathrm{L}^{-1}\right)$, respectively.

The initial and boundaries conditions are:

$t=0 \rightarrow C(z, 0)=0$

$z=0 \rightarrow C=C_{0}+\frac{D_{L} \cdot \varepsilon}{v_{z}} \cdot \frac{\partial C}{\partial z}$

$z=H \rightarrow \frac{\partial C}{\partial z}=0$

The differential mass balance in the solid phase, assuming that the adsorption system can be explained by the Langmuir model, conform:

$\frac{\partial q}{\partial t}=k_{1} \cdot C \cdot\left(q_{\max }-q\right)-k_{2} \cdot q$

where $q_{\max }$ is the maximum adsorption capacity of the resin $\left(\mathrm{mg} \mathrm{L}^{-1}\right), k_{1}$ and $k_{2}$ are intrinsic kinetics constants $\left(\mathrm{L} \mathrm{mg}^{-1} \mathrm{~h}^{-1}\right.$ and $\mathrm{h}^{-1}$, respectively) and $q$ is the CPC concentration adsorbed on the resin $\left(\mathrm{mg} \mathrm{L}^{-1}\right)$.

The initial condition is:

$t=0 \rightarrow q(z, 0)=0$

Considering that the rate of adsorption of the product into the resin is equal to the decreasing concentration of the product in the bulk phase, Eqs. (1) and (5) could be manipulated to explicit

Table 2

Experimental and predicted column efficiency for the CPC adsorption in the fixed-bed column.

\begin{tabular}{|c|c|c|c|c|c|c|c|c|}
\hline Run & $v_{z}\left(\mathrm{~m} \mathrm{~h}^{-1}\right)$ & $L(\mathrm{~m})$ & $d_{p}(\mu \mathrm{m})$ & $\Phi_{90 \%}^{\exp }(\%)$ & $\Phi_{90 \%}^{\text {calc }}(\%)$ & $\operatorname{RED}(\%) \operatorname{RED}=$ & $\frac{\Phi_{90 \%}^{\exp }-\Phi_{90 \%}^{\text {calc }}}{\Phi_{90 \%}^{\exp }}$ & $\cdot 100$ \\
\hline 1 & 0.306 & 0.075 & 297.0 & 55.8 & 58.5 & 4.8 & & \\
\hline 2 & 0.306 & 0.10 & 297.0 & 59.5 & 64.0 & 7.6 & & \\
\hline 3 & 0.306 & 0.05 & 210.0 & 62.8 & 64.5 & 2.7 & & \\
\hline 4 & 0.306 & 0.05 & 500.0 & 41.9 & 45.9 & 9.5 & & \\
\hline 5 & 0.153 & 0.05 & 297.0 & 58.5 & 64.4 & 10.1 & & \\
\hline 6 & 0.612 & 0.05 & 297.0 & 41.7 & 45.1 & 8.2 & & \\
\hline $7^{a}$ & 0.306 & 0.05 & 297.0 & 50.1 & 51.1 & 2.0 & & \\
\hline
\end{tabular}

a Validation. 
the concentration of CPC adsorbed on the surface particle $\left(C_{r=R_{p}}\right)$ conform:

$\frac{\partial q}{\partial t}=\left(\frac{1-\varepsilon}{\varepsilon}\right) \cdot \frac{3 \cdot k_{f}}{R_{p}} \cdot\left(C-C_{r=R_{p}}\right)=k_{1} \cdot C \cdot\left(q_{\max }-q\right)-k_{2} \cdot q$

After the algebraic manipulations the concentration of CPC adsorbed on the surface particle is:

$C_{r=R_{p}}=\frac{C+\left(R_{p} k_{2} q / 3 k_{f}\right)(1-\varepsilon / \varepsilon)}{1+\left[\left(k_{1} R_{p} / 3 k_{f}\right)\left(q_{\max }-q\right)\right](1-\varepsilon / \varepsilon)}$

To solve the partial differential equation presented in Eq. (1), the time and spatial derivatives were solved by the Crank-Nicholson approximations, resulting in a tri-diagonal linear system, which was solved by the DLSLRG IMSL FORTRAN routine. The differential mass balance in the solid phase (Eq. (5)) was integrated in the time domain using the LIMEX routine for each column positions [17]. The model parameters, namely $q_{\max }, k_{1}, k_{2}, D_{L} k_{f}$, and $\varepsilon$ were estimated by the Particle Swarm Optimization (PSO). An in-house algorithm, developed in our working group, was used and implemented in FORTRAN 90 language.

A common practice in the modeling of adsorption processes is the use of kinetic parameters $\left(k_{1}\right.$, and $\left.k_{2}\right)$ and equilibrium parameter capacity ( $q_{\max }$ ) estimated by nonlinear regression, besides the empirical correlations to calculate the transport parameters $\left(D_{L}\right.$ and $k_{f}$ ) and bed properties (porosity) in order to simulate the process in a fixed-bed or expanded-bed column. Nevertheless, these correlations are used to obtain these parameters in a more general application, where its value is valid for a narrow range of operational variables. In this sense, it is preferred to estimate from experimental data.

The parameters estimation consisted of minimizing the sum squares residues (SSR), as described in Eq. (9), where $C$ is the experimentally CPC concentration at $z=H$ and $C^{\text {calc }}$ is the calculated CPC concentration at $z=H$ by the model, both normalized between 0 and 1.

$\mathrm{SSR}=\sum_{i=1}^{N}\left(C_{i}-C_{i}^{\text {calc }}\right)^{2}$

For the estimation of the parameters were employed six breakthrough curves, using a fixed initial CPC concentration of $60.0 \mathrm{mg} \mathrm{L}^{-1}$. The variables evaluated on the performance of the process were column length, particle diameter and superficial velocity. In the validation step, one additional breakthrough curve was employed. Table 2 presents the detailed information about the experimental conditions used in the seven breakthrough curves.

\subsection{Particle Swarm Optimization (PSO)}

The PSO version used in this study was based on the work of Schwaab et al. [7], which presents a detailed description of the algorithm. The PSO technique is based on the social behavior of collection of animals. Each individual of the swarm, called particle, remembers the best solution found by itself and by the whole swarm along the search trajectory. The particles move along the search space and exchange information with others particles, in accordance with the following equations:

$v_{p, d}^{k+1}=w \cdot v_{p, d}^{k}+c_{1} \cdot r_{1}\left(x_{p, d}^{\text {ind }}-x_{p, d}^{k}\right)+c_{2} \cdot r_{2}\left(x_{d}^{\text {glob }}-x_{p, d}^{k}\right)$

$x_{p, d}^{k+1}=x_{p, d}^{k}+v_{p, d}^{k+1}$

In Eqs. (10) and (11), $p$ denotes the particle, $d$ is the search direction, $k$ represents the iteration number, $v$ is the velocity (or pseudovelocity) of the particle and $x$ is the position of particle, $x^{\text {ind }}$ and $x^{\text {glob }}$ represent the regions of the search space where the objective function attains low (optimum) values, where $x^{\text {ind }}$ is the best position found by the particle itself, while $x^{\text {glob }}$ is the best position found by whole swarm. In addition, $r_{1}$ and $r_{2}$ are two random numbers with uniform distribution in the range comprehended between 0 and 1 . The parameters $w, c_{1}$ and $c_{2}$ are search parameters, which are called of inertial weight, the cognition and social parameters, respectively.

After a preliminary set of simulations, it was defined the best configuration of the PSO algorithm that is as following: 40 particles were used for the PSO algorithm, and the inertial weight, cognition and social parameters were set at $0.7,1.0,1.0$, respectively. The search interval for model parameters was determined according the studies available in the literature for the separation of CPC and was allowed to vary as following: $q_{\max } \in[0.1,100]$, $k_{1}, k_{2} \in\left[1 \times 10^{-4}, 0.1\right], D_{l} \in\left[1 \times 10^{-8}, 1 \times 10^{-3}\right], k_{f} \in[0.1,20]$, and $\varepsilon \in[0.20,0.55]$.

The proceeding of minimization of the objective function was repeated four times, since the $\mathrm{PSO}$ is a heuristic method of optimization and do not guarantee to find the minimum global. After each minimization of the objective function the parameters range were decreased, with objective to guarantee that the region of minimum global was found.

\subsection{Process optimization}

The main advantage of a mathematical model is that it can in principle be used to simulate the process at other experimental conditions than those used to estimate the model parameters. In this section, an experimental design (central composite rotational design - CCRD) was conceived taking into account the effects of column length and superficial velocity as process variables with the responses (column efficiency - Eq. (12), and breakthrough time $\left.-t_{C / C_{0}=0.9}\right)$ obtained from the application of the validated model keeping fixed the resin diameter at $210 \mu \mathrm{m}$. In this sense, the hybrid model works at a process simulator affording a process answer from a given input. The column efficiency was defined according the following equation [11]:

$\Phi_{90 \%}=\frac{\left(0.9 \cdot t_{C / C_{0}=0.9}-\int_{0}^{. t_{C / C_{0}=0.9}} C / C_{0} \cdot d t\right)}{0.9 \cdot t_{C / C_{0}=0.9}} \cdot 100$

In the fixed-bed adsorption process the column efficiency and breakthrough time had opposite tendency, since as high as the breakthrough time more efficient is the column. However, there is an interval for the independent variables that both, column efficiency and breakthrough time, are at their optimum values. At this point, the process reached its operational equilibrium and to find this point it was defined a third response, which is function of column efficiency and breakthrough time. This new response is defined as the objective function conforms following equation:

$F=\frac{\overline{\Phi_{90 \%}}+\overline{\Phi_{90 \%}^{2}}+\overline{\Phi_{90 \%}^{3}}}{1+\bar{\Theta}+\overline{\Theta^{2}}+\overline{\Theta^{3}}}$

where $\overline{\Phi_{90 \%}}$ and $\bar{\Theta}$ are the column efficiency and breakthrough time normalized between 0 and 1 . The optimization problem was stated as the maximization of $F$ in function of superficial velocity and column length so that the feasibility of the purification process was dependent of the compromise between high efficiency and low required time. The responses in terms of column efficiency, breakthrough time and F were analyzed by Statistica ${ }^{\circledR} 7.0$ Statsoft Inc. 


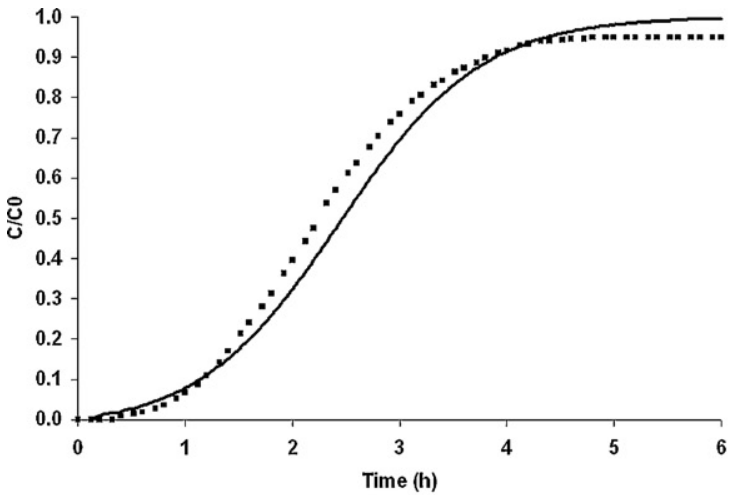

$\operatorname{Exp} 1$

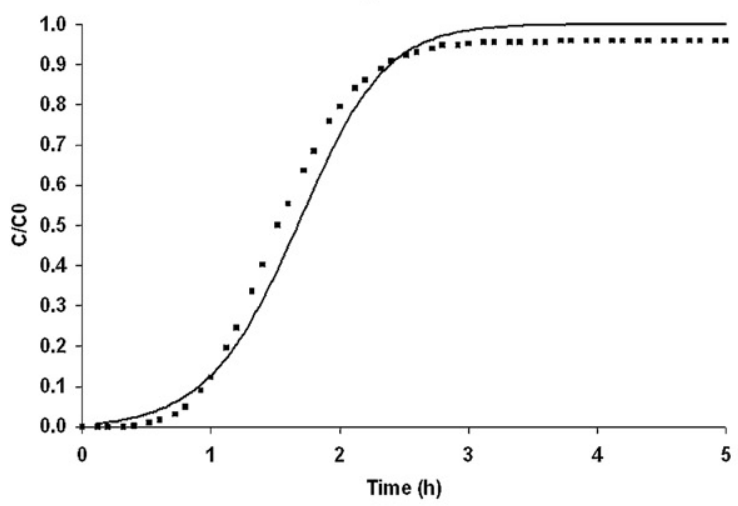

$\operatorname{Exp} 3$

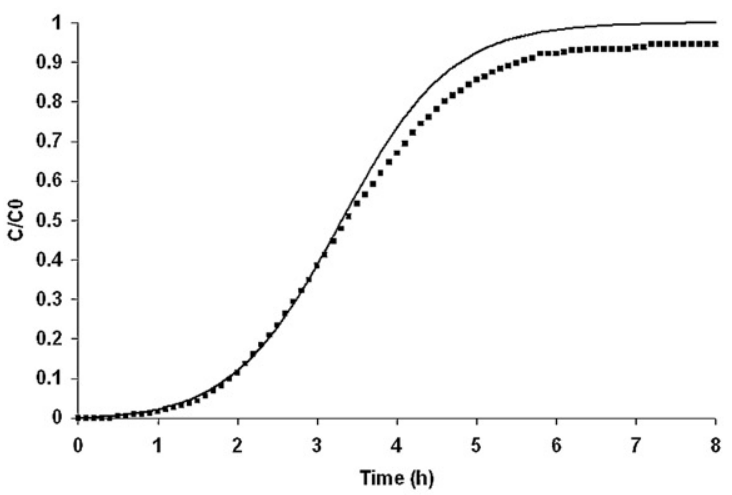

$\operatorname{Exp} 5$

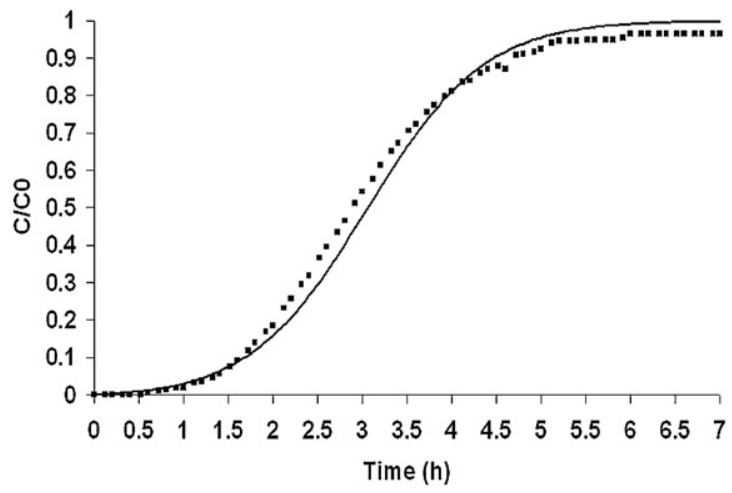

$\operatorname{Exp} 2$

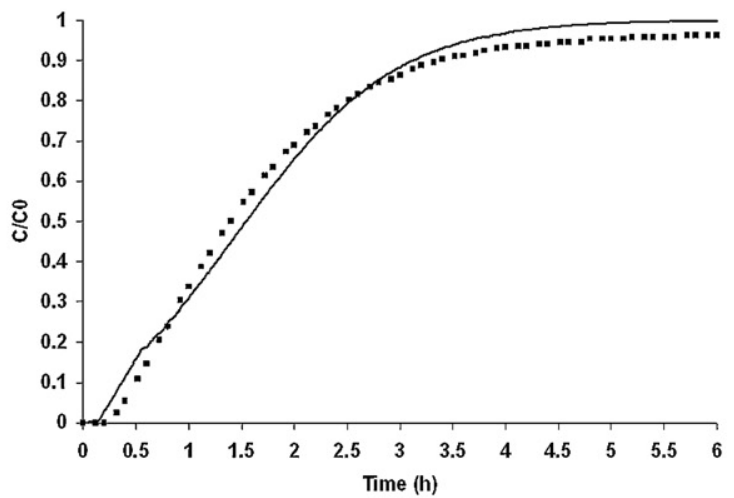

$\operatorname{Exp} 4$

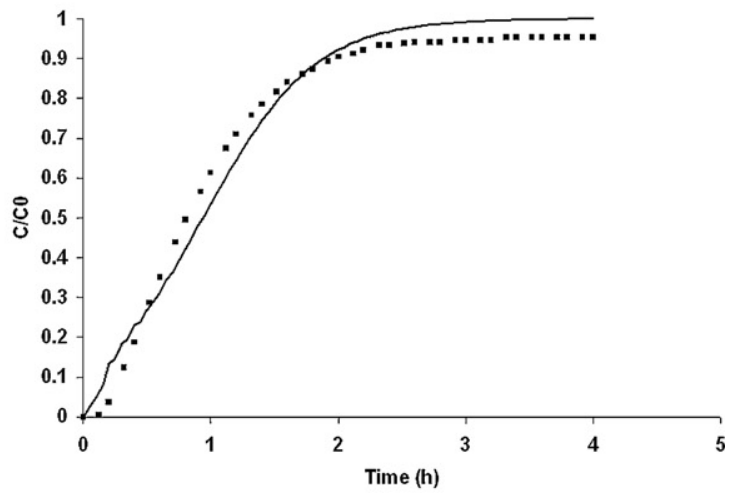

$\operatorname{Exp} 6$

Fig. 1. Experimental and predicted breakthrough curves for CPC adsorption in the fixed-bed column employed during the parameter estimation procedure.

\section{Results and discussion}

\subsection{Experimental breakthrough curves and column efficiency}

Table 2 presents the column efficiency calculated by the experimental breakthrough curves according to Eq. (10). The highest efficiency was $62.8 \%$ on run 3 and the lowest efficiencies were reported at runs 4 and 6 , which were 41.9 and $41.7 \%$, respectively. The effect of particle size in the column efficiency is clearly demonstrated at runs 3 and 4 . The increase in the particle diameter from 297 to $500 \mu \mathrm{m}$ decreased the column efficiency. As big as the particle size the contact area between CPC and resin is considerably reduced, limiting the efficiency of the process, since the diffusion process was not taken into account in this work.

The superficial velocity is another important parameter that has impact on the efficiency. Its effect can be better visualized at runs
5, 6 and 7. At constant column length and particle size, the gradual increase in the superficial velocity results in a decrease of the column efficiency. This occurs because at high velocities the contact time between CPC and the resin is lower, affecting the dynamic capacity, that have direct influence on the adsorption kinetics. The contact time can be increased by increasing the column length. Runs 1, 2 and 7 illustrate the effect of column length on efficiency at constant velocity and particle size. Higher efficiencies are obtained with higher column lengths.

Another important aspect that should be carefully observed in adsorption purification process is the breakthrough time. The feasibility of the purification process is dependent of the compromise between high efficiency and low required time, which should be determined. The experimental breakthrough time obtained in this work ranged from $1.9 \mathrm{~h}$ for run 6 to $4.8 \mathrm{~h}$ for run 5 (Fig. 1). An example that there is an optimum relationship between break- 
through time and efficiency can be verified at runs $3(2.4 \mathrm{~h})$ and 6 $(1.9 \mathrm{~h})$. The efficiency at run 3 was $20 \%$ higher than run 6 . However, the breakthrough time obtained in run 3 was only 30 min higher than run 6 . Nevertheless, the efficiency should be improved and, in the following section (Section 4.3), it was presented a tool, based on mathematical model of the process, to adjust the operational parameters (column length and superficial velocity) to maximize the efficiency and minimize the breakthrough time.

\subsection{Parameter estimation and model validation}

Model parameters were estimated by the Particle Swarm Optimization (PSO) algorithm using a set of six experimental breakthrough curves at different conditions (Table 2). It is important emphasize that this study did not compare PSO to other parameter estimation methods and that techniques such as genetic algorithms, simulated annealing, ant algorithm too could in principle provide satisfactory parameter estimates for this particular problem. The estimated parameters are presented at Table 3.

A numerical comparison of the estimated parameters obtained in this work with the ones reported in the literature is difficult for two reasons. Firstly, the units differ amongst the works and a simple unit conversion can lead to mistakes of interpretation, because the conversions alter their magnitude and these new values may not be the optimum. Additionally, the strategy of global parameter estimation adopted in this work was not employed previously to simulate adsorption process. Several works report the use of a set of parameters for each experimental condition, which is a different strategy that adopted in this study $[3-5,18]$.

The model parameters in Table 3 were used to simulate the CPC adsorption process and results are showed at Fig. 1 . As it can be seen, the model was able to simulate the CPC breakthrough curves in all the experiments. Some deviations were observed, mainly in the final stage of the breakthrough curves. The reliability and applicability of the proposed model to simulate the breakthrough curves of CPC in the fixed-bed column was verified in terms of the column efficiency (Eq. (10)). Table 2 presents the experimental and calculated column efficiencies, which emphasizes that model predictions are reliable, since the relative error deviations were lower than $10 \%$ in all the experiments.

The critical test for the validity of a model is not its ability to fit a given condition. Rather, it is the ability of the model to predict a given set of conditions that constitute the true test of the validity of the model, its assumptions and the estimated parameter values [19]. In this work one additional experimental breakthrough curve was used to validate the model, whose operational conditions are detailed at run 7, in Table 2. The results are shown in Fig. 2, where it can be verified that the proposed model well fitted the CPC breakthrough curve. In addition, Table 2 presents the experimental and calculated column efficiencies for the validation experiment (run 7 ). The relative error deviation was the lowest amongst the experiments. This fit quality shows that the estimated parameters and the hypothesis are valid and can be used to simulate the CPC adsorption in other conditions, different of those employed during the parameter estimation.

According Schwaab and Pinto [20] the variance of model could be represented by the following equation:

$\hat{\sigma}^{2}=\frac{\mathrm{SSR}}{N P-N}$

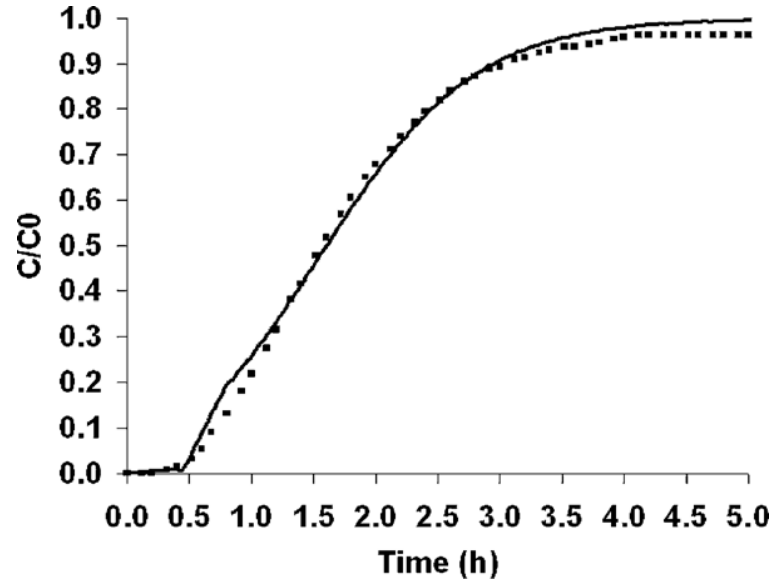

Fig. 2. Experimental and predicted breakthrough curves for $\triangle P C$ adsorption in the fixed-bed column employed during the model validation.

Table 4

Parametric sensitivity of the model parameters.

\begin{tabular}{lll}
\hline Parameters & \multicolumn{2}{c}{$\Phi_{90 \%}^{\text {calc }}(\%)$} \\
\cline { 2 - 3 } & $-15 \%$ & $+15 \%$ \\
\hline$q_{\max }\left(\mathrm{mg} \mathrm{L}^{-1}\right)$ & 46.7 & 52.1 \\
$k_{1}\left(\mathrm{~L} \mathrm{mg}^{-1} \mathrm{~h}^{-1}\right)$ & 92.4 & 92.4 \\
$k_{2}\left(\mathrm{~h}^{-1}\right)$ & 49.9 & 49.2 \\
$D_{1}\left(\mathrm{~m}^{2} \mathrm{~h}^{-1}\right)$ & 49.5 & 49.5 \\
$k_{f}\left(\mathrm{~m} \mathrm{~h}^{-1}\right)$ & 49.5 & 49.5 \\
$\varepsilon$ & 57.9 & 42.6 \\
\hline
\end{tabular}

where $\hat{\sigma}^{2}$ is the variance of model predictions, SSR is the sum of square residues, $N P$ is the number of models parameters and $N$ is the number of experimental points. If the models predictions are reliable, the experimental variance is comparable to the variance of the model. In this work, the experimental variance was 0.1277 , while the variance of model predictions was 0.1434 . Performing the test of Fisher it is seen that these variances are statistically similar $(p<0.05)$, indicating that the model predictions are reliable.

In order to quantify the sensitivity of the model predictions, with respect to errors in the estimated parameters, a sensitivity analysis has been performed. The parameters presented at Table 3 were taken as nominal values. The process model was solved with a relative parameter perturbation ranging from -15 to $+15 \%$ in relation to nominal values. To quantify the deviation occasioned by the parameter perturbation, the column efficiency was calculated according Eq. (10) for the validation experiment (run 7) and comparing with nominal value (51.1\%). As it is shown in Table 4, the most sensitive variable was the intrinsic constant $k_{1}$. The alteration in its value leads to an accentuated variation in the column efficiency. The bed porosity was the second most sensitive variable in the process. The increase in the porosity decreases the efficiency, because there is an increase in the void space of the column. In practice, there is a loss of dynamic binding capacity, because there are lower molecules of $\mathrm{CPC}$ in contact with the resin.

When the model predictions are very sensitive to a parameter value, like $k_{1}$, it means that the confidence interval of this parameter will be very narrow and this parameter can be estimated with high precision. In fact, when the sensitivity of a parameter is very low, the estimation of this value is very difficult, since the change of the

Table 3

The estimated parameters by the PSO algorithm.

\begin{tabular}{|c|c|c|c|c|c|c|}
\hline Parameters & $q_{\max }\left(\mathrm{mg} \mathrm{L}^{-1}\right)$ & $k_{1} \times 10^{2}\left(\mathrm{~L} \mathrm{mg}^{-1} \mathrm{~h}^{-1}\right)$ & $k_{2}\left(\mathrm{~h}^{-1}\right)$ & $D_{\mathrm{l}} \times 10^{5}\left(\mathrm{~m}^{2} \mathrm{~h}^{-1}\right)$ & $k_{f}\left(\mathrm{~m} \mathrm{~h}^{-1}\right)$ & $\varepsilon$ \\
\hline Values & 87.44 & 2.40 & 0.67 & 1.39 & 7.64 & 0.25 \\
\hline
\end{tabular}


Table 5

Matrix of the CCRD (real and coded values) with responses in terms of the column efficiency, breakthrough time and objective function for CPC purification in a fixedbed column.

\begin{tabular}{llllrl}
\hline Simulation & $v_{z}\left(\mathrm{~m} \mathrm{~h}^{-1}\right)$ & $L(\mathrm{~m})$ & $\Phi_{90 \%}^{\text {calc }}(\%)$ & \multicolumn{1}{c}{$t_{C / C_{0}=0.9}(\mathrm{~h})$} & \multicolumn{1}{l}{$F$} \\
\hline 1 & $0.19(-1)$ & $0.16(-1)$ & 81.82 & 9.84 & 1.14 \\
2 & $0.61(1)$ & $0.16(-1)$ & 59.98 & 3.95 & 0.03 \\
3 & $0.19(-1)$ & $0.44(1)$ & 92.62 & 24.44 & 1.09 \\
4 & $0.61(1)$ & $0.44(1)$ & 79.61 & 8.44 & 1.02 \\
5 & $0.10(-1.41)$ & $0.30(0)$ & 94.13 & 31.20 & 0.82 \\
6 & $0.7(1.41)$ & $0.30(0)$ & 70.21 & 5.20 & 0.46 \\
7 & $0.40(0)$ & $0.10(-1.41)$ & 58.90 & 3.81 & 0.00 \\
8 & $0.40(0)$ & $0.50(1.41)$ & 87.11 & 13.90 & 1.37 \\
9 & $0.40(0)$ & $0.30(0)$ & 80.17 & 8.94 & 1.04 \\
\hline
\end{tabular}

parameter value produces only a small modification in the sum of squared residues, turning hard the estimation of this parameter. In this work the PSO algorithm has shown to be a suitable method for parameter estimation in adsorption processes since it overcomes the difficulties imposed by the high parameter sensitivity.

\subsection{Optimization of column efficiency}

Table 5 presents the results obtained in the CCRD for column efficiency, breakthrough time and objective function $(F)$. The efficiency ranged from $58.90 \%$ to $94.13 \%$ and the breakthrough time from 3.81 to $31.20 \mathrm{~h}$ at simulations 7 and 5, respectively. The values of $F$ ranged from 0 to 1.37 at simulations 7 and 8, respectively. As expected, the highest efficiencies were obtained at high column length and low superficial velocity. However, a more profound analysis only can be made by the effects of each variable in the response.

The data in Table 5 were used to compute the linear, quadratic and interaction effects of the process variables in the efficiency,
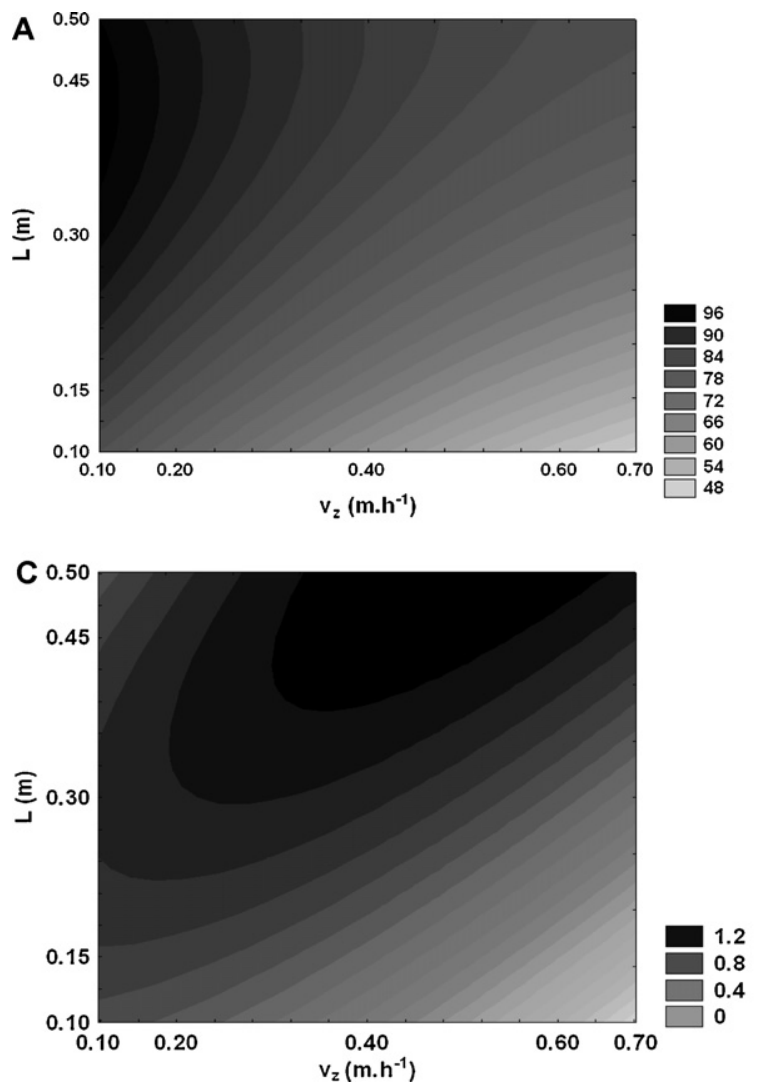

breakthrough time and $\mathrm{F}$, which are presented in Table 6 . The significant effects $(p<0.15)$ in the column efficiency were the linear and quadratic terms for column length, the linear term for superficial velocity and the interaction term, while for breakthrough time the most significant effects were the linear term for column length and the linear and quadratic effects for superficial velocity. The increase of the superficial velocity shows a negative effect in both column efficiency and breakthrough time, while the column length shows positive effect. However, the magnitude of the column length effect in efficiency and breakthrough time is different, since the effect was more pronounced in efficiency.

By the analysis of the effects it was possible to verify that the efficiency is directly proportional to column length and inversely proportional to superficial velocity, while the breakthrough time had opposite behavior. To found an optimum point that presents high efficiency and low breakthrough time is a difficult and timeconsuming task, mainly if the traditional one-variable-at-a-time approach is employed. Response surface methodology (RSM) is a scientific approach to determine optimum conditions, which combines special experimental designs with Taylor first and second order equations. The RSM protocol, therefore, uses the Taylor equation to approximate the function, which describes the response in nature, coupled with the special experimental designs for determining the coefficients of the Taylor equation that is employed to draw the contour plots and response surface [20-22].

The quadratics coded models that better describes the responses in Table 6 (column efficiency, breakthrough time and $F$ ) were validated by analysis of variance (ANOVA) and were employed to generate contour plots. The fitted contour plots for column efficiency, breakthrough time and F were generated using Statistica 7.0 and are presented in Fig. 3. The highest efficiency was obtained for column length ranging from 0.30 to $0.50 \mathrm{~m}$ (96\%) at low level of superficial velocity $\left(0.10 \mathrm{~m} \mathrm{~h}^{-1}\right)$. An increment in the superficial

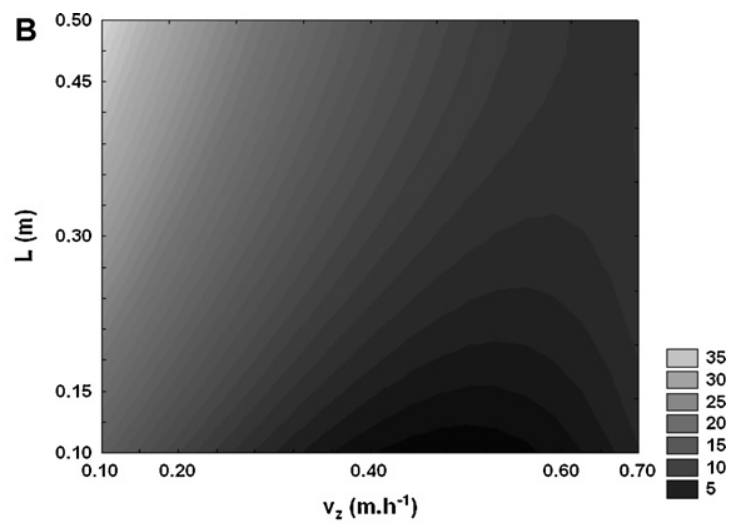

Fig. 3. Contour plots for column efficiency (A), breakthrough time (B) and objective function (C) for CPC purification in the fixed-bed column. 
Table 6

Estimated effects of manipulated variables on column efficiency and breakthrough time for CPC purification.

\begin{tabular}{|c|c|c|c|c|c|c|c|c|c|}
\hline & \multicolumn{3}{|c|}{ Column efficiency } & \multicolumn{3}{|c|}{ Breakthrough time } & \multicolumn{3}{|l|}{$F$} \\
\hline & Effects & Standard error & $p$-Value & Effects & Standard error & $p$-Value & Effects & Standard error & $p$-Value \\
\hline Mean & 80.16 & 2.10 & $<0.0001$ & 8.96 & 3.56 & 0.0861 & 1.04 & 0.28 & 0.0335 \\
\hline$v_{z}$ & -17.20 & 1.49 & 0.0014 & -14.68 & 2.52 & 0.0101 & -0.42 & 0.20 & 0.1209 \\
\hline$L$ & 17.60 & 1.49 & 0.0013 & 8.35 & 2.52 & 0.0451 & 0.72 & 0.33 & 0.0353 \\
\hline$v_{z}^{2}$ & 2.49 & 2.48 & 0.3882 & 8.34 & 4.18 & 0.1401 & -0.32 & 0.20 & 0.4000 \\
\hline$L^{2}$ & -6.73 & 2.48 & 0.0728 & -1.06 & 4.18 & 0.8168 & -0.27 & 0.33 & 0.4626 \\
\hline$v_{z} \times L$ & 4.42 & 2.11 & 0.1269 & -5.06 & 3.56 & 0.2503 & 0.52 & 0.28 & 0.1583 \\
\hline
\end{tabular}

velocity value decreases the column efficiency (Fig. 3A). Regarding the breakthrough time, an opposite behavior was found, since at conditions where the column efficiency is maximized the breakthrough time is too large (Fig. 3B). Both column efficiency and breakthrough time showed behavior already expected.

The greatest advantage of the response surface methodology in optimizing two or more responses is the easy visualization of optimum region for both responses. In this work, the two responses investigated (column efficiency and breakthrough time) showed opposite behavior, as previously discussed. Nevertheless, there are regions in Fig. $3 \mathrm{C}$ that make it possible to obtain high efficiency at acceptable breakthrough time, since the $\mathrm{F}$ values were the greatest. The predicted efficiency for a column length of $0.50 \mathrm{~m}$ and superficial velocity of $0.32 \mathrm{~m} \mathrm{~h}^{-1}$ was about $90 \%$ and the breakthrough time was around $15 \mathrm{~h}$. At these conditions the column efficiency decreased $6 \%$, which is undesirable but reasonable, and $57 \%$ for breakthrough time, which is highly positive.

\section{Conclusion}

In this work, the mathematical model of the fixed-bed adsorption process for CPC purification was described. The PSO algorithm was used to optimize the model parameters and proved to be an efficient method for parameter estimation for adsorption processes. Modeling and simulation of CPC purification showed a good performance during both estimation and validation step. The use of experimental design methodology to plan the experiments coupled with the validate model as a process simulator showed to be an interesting alternative for process optimization, since the optimum was found quickly and with few simulations. The proposed methodology saves time and reduces costs in adsorption process development and scale-up.

\section{Acknowledgements}

The authors are grateful to CAPES, CNPq and FEA/UNICAMP for financial support of this work and scholarships.

\section{Appendix A. Nomenclature}

C CPC concentration in bulk-phase $\left(\mathrm{mg} \mathrm{L}^{-1}\right)$

$C_{r=R_{p}} \quad$ CPC concentration on resin surface $\left(\mathrm{mg} \mathrm{L}^{-1}\right)$

$C_{0}$ initial CPC concentration at the inlet of the column $\left(\mathrm{mg} \mathrm{L}^{-1}\right)$

$C_{i} \quad$ experimental CPC concentration normalized between 0 and $1(-)$

$C_{i}^{\text {calc }} \quad$ calculated CPC concentration normalized between 0 and $1(-)$

$D_{L} \quad$ axial dispersion coefficient $\left(\mathrm{m}^{2} \mathrm{~h}^{-1}\right)$

$F \quad$ objective function for process optimization (-)

$H \quad$ column height $(\mathrm{m})$

$k_{1} \quad$ intrinsic adsorption constant $\left(\mathrm{L} \mathrm{mg}^{-1} \mathrm{~h}^{-1}\right)$

$k_{2} \quad$ desorption constant $\left(\mathrm{h}^{-1}\right)$ $k_{f} \quad$ liquid film mass transfer coefficient $\left(\mathrm{m} \mathrm{h}^{-1}\right)$

$N \quad$ number of experimental data points (-)

NP number of model parameters (-)

$q \quad$ adsorbed CPC concentration ( $\mathrm{mg} \mathrm{L}^{-1}$ of adsorbent)

$q_{\max } \quad$ maximum adsorption capacity ( $\mathrm{mg} \mathrm{L}^{-1}$ of adsorbent)

$R_{p} \quad$ particle radius (m)

SSR sum squares residues (-)

$t$ time (h)

$v_{z} \quad$ superficial liquid velocity $\left(\mathrm{m} \mathrm{h}^{-1}\right)$

$z \quad$ axial bed height $(\mathrm{m})$

Nomenclature for Eqs. (10) and (11), referring the PSO method

$c_{1} \quad$ search parameter denominated the cognition parameter

$c_{2}$ search parameter denominated social parameter

$d$ the search direction

$k \quad$ the iteration number

$p \quad$ denotes the particle

$r_{1}$ and $r_{2}$ random numbers with uniform distribution in the range $[0,1]$

$v \quad$ velocity (or pseudo-velocity) of the particle and

$w \quad$ search parameters denominated inertial weight

$x \quad$ position of particle

$x^{\text {glob }} \quad$ the best position found by whole swarm

$x^{\text {ind }} \quad$ the best position found by the particle itself

\section{Greek letters}

$\varepsilon \quad$ bed voidage

$\Phi_{90 \%} \quad$ column efficiency (\%)

$\overline{\Phi_{90 \%}} \quad$ column efficiency normalized between 0 and $1(-)$

$\bar{\Theta} \quad$ breakthrough time normalized between 0 and $1(-)$

$\hat{\sigma} \quad$ variance of model predictions

\section{References}

[1] Bautista LF, Casillas JL, Martínez M, Aracil J. Functionalized adsorbents for the purification of cephalosporin C and deacetylcephalosporin C. Ind Eng Chem Res 2006;45:3230-6.

[2] Ramos AM, Otero M, Rodrigues AE. Recovery of vitamin B12 and cephalosporin$C$ from aqueous solutions by adsorption on non-ionic polymeric adsorbents. Sep Purif Technol 2004;28:85-98.

[3] Lee JW, Jung HJ, Moon H. Effect of operating conditions on adsorption of cephalosporin C in a column adsorber. Korean J Chem Eng 1997;14: 277-84.

[4] Lee JW, Park HC, Moon H. Adsorption and desorption of cephalosporin C on nonionic polymeric sorbents. Sep Purif Technol 1997;12:1-11.

[5] Yang SA, Pyle DL. The adsorption kinetics of cephalosporin-c on non-ionic polymeric macropore Amberlite XAD-16 resin. J Chem Technol Biotechnol 1999;74:216-9.

[6] Kennedy J, Eberhart R. Particle swarm optimization. In: Proceedings of the IEEE International Conference on Neural Networks. 1948. p. 1942-8.

[7] Schwaab M, Biscaia EC, Monteiro JL, Pinto JC. Nonlinear parameter estimation through particle swarm optimization. Chem Eng Sci 2008;63:1542-8.

[8] Eftaxias A, Font J, Fortuny A, Fabregat A, Stuber F. Nonlinear kinetic parameter estimation using simulated annealing. Comput Chem Eng 2002;26:1725-33.

[9] Razavi S, Jalali-Farahani F. Optimization and parameter estimation in petroleum engineering problems using ant colony algorithm. J Petrol Sci Eng 2010;74:147-53.

[10] Costa CBB, Rivera EAC, Rezende MCAF, Maciel MRW, Maciel Filho R. Prior detection of genetic algorithm significant parameters: coupling factorial design technique to genetic algorithm. Chem Eng Sci 2007;62:4780-801. 
[11] Moraes CC, Mazutti MA, Rodrigues MI, Maugeri F, Kalil SJ. Mathematical modeling and simulation of inulinase adsorption in expanded bed column. J Chromatogr A 2009;1216:4395-401.

[12] Fiorentin LD, Trigueros DEG, Módenes AN, Espinoza-Quiñones FR, Pereira NC, Barros STD, et al. Biosorption of reactive blue $5 \mathrm{G}$ dye onto drying Orange bagasse in batch system: kinetic and equilibrium modeling. Chem Eng J 2010;163:68-77.

[13] Kuhn RC, Purification of fructooligosaccharides obtained trhough enzymatic synthesis using fixed-bed columns and nanofiltration membranes. Ph.D. Thesis, Campinas, Brazil: University of Campinas; 2010.

[14] Salto F, Prieto JG. Interactions of and penicillins with nonpolar macroporous styrenedivinylbenzene copolymers. J Pharm Sci 1981;70:994-8.

[15] Kirkby NF, Slater NKH, Weisenberger KH, Addo-Yobo F, Doulia D. An HPLC technique for parameter estimation for reversed-phase chromatography: a case study on cephalosporin C. Chem Eng Sci 1986;41:2005-16.

[16] Perrin DD. Buffers of low ionic strength for spectrophotometric pK determination. Aust J Chem 1963;16:572-8.
[17] Deuflhard E, Hairer E, Zugck J. One step and extrapolation methods for differential-algebraic systems. Numer Math 1987;51:501-16.

[18] Barboza M, Hokka CO, Maugeri F. Continuous cephalosporin C purification: dynamic modeling and parameter validation. Bioprocess Biosyst Eng 2002;25:193-203.

[19] Firouztale E, Maikner JJ, Deissler KC, Cartier PG. Validation of a theoretical model for adsorption using cephalosporin $C$ and polymeric reversed-phase resins. J Chromatogr A 1994;658:361-70.

[20] Schwaab M, Pinto JC. Análise de dados experimentais I: Fundamentos de estatística e estimação de parâmetros. e-Papers, Rio de Janeiro; 2007.

[21] Haaland PD. Experimental design in biotechnology. New York: Marcel Decker Inc.; 1989.

[22] Rodrigues MI, Iemma AF. Planejamento de Experimentos e Otimização de Processos: Uma estratégia seqüencial de planejamentos. Campinas: Casa do Pão; 2005. 